Technical Note

\title{
Evaluation of jaws-only intensity modulated radiation therapy treatment plans using Octavius 4D system
}

\author{
Duong THANH TAl ${ }^{1,2,4, a}$, Luong Thi OANH ${ }^{2,4}$, Nguyen Dong SON ${ }^{3}$, Truong Thi Hong LOAN ${ }^{2}$ \\ ${ }^{I}$ Department of Radiation Oncology, Dong Nai General Hospital, Bien Hoa, 810000, Vietnam \\ ${ }^{2}$ Faculty of Physics \& Engineering Physics, VNUHCM - University of Science, Ho Chi Minh, 749000, Vietnam \\ ${ }^{3}$ Chi Anh Medical Technology Co.,Ltd., Ho Chi Minh, 717066, Vietnam \\ ${ }^{4}$ Faculty of Medicine, Nguyen Tat Thanh University, Ho Chi Minh, 702000, Vietnam \\ ${ }^{a}$ E-mail address: thanhtai_phys@yahoo.com
}

(received 1 December 2017; revised 23 February 2018, 12 April 2018; accepted 18 April 2018)

\begin{abstract}
Introduction: Jaws-Only Intensity modulated radiation therapy (JO-IMRT) is a technique uses the collimator jaws of the linear accelerator (LINAC) to delivery of complex intensity patterns. In previous studies, pretreatment patient specific quality assurance for those JO-IMRT were also performed using ionization chamber, MapCHECK2, and Octavius 4D and good agreements were shown. The aim of this study is to further verify JO-IMRT plans in 2 different cases: one with the gantry angle set equal to beam angle as in the plans and the other with gantry angle set to zero degree.

Materials and Methods: Twenty-five JO-IMRT, previously verified, were executed twice for each plan. The first one used a real gantry angle, and the second one used a $0^{\circ}$ gantry angle. Measurements were performed using Octavius 4D 1500.

Results: The results were analyzed using Verisoft software. The results show that the Gamma average was 97.32 $\pm 2.21 \%$ for IMRT with a $0^{\circ}$ gantry angle and $94.72 \pm 2.67 \%$ for IMRT with a true gantry angle.

Conclusion: In both cases, gamma index of more than $90 \%$ were found for all of our 25 JO-IMRT treatment plans.
\end{abstract}

Key words: intensity-modulated radiation therapy (IMRT); jaws only IMRT (JO-IMRT); quality assurance (QA); Octavius 4D; MapCHECK2.

\section{Introduction}

Cancer is one of the most serious diseases, which affect many people, and is the most common causes of death in the word. Radiation therapy plays an important role in treating cancer. The goal of radiation therapy is twofold: maximize the possibility of destroying malignant cells while minimizing the damage to healthy tissue. The introduction of intensity modulated radiation therapy (IMRT) technique has brought improvements in this goal [1]. In most oncology centers, this technique is facilitated by the use of multi-leaf collimators (MLC) which is the main tool for beam shaping on the linear accelerators (LINAC). The MLC is a complicated device that is generally make of tungsten leaves and controlled by many motors [2,3]. Otherwise, MLC has also some factors disadvantages such as radiation leaf leakage, tongue and groove effect, and backscattered radiation into the machine monitor chamber [3-6]. Meanwhile, several oncology centers in developing countries are still using cobalt machines or linear accelerators (LINAC) without MLCs which are expensive acquisition costs, not yet available and some disadvantage. From the fact that, some investigators also intend to explore the use of independent jaws only instead of MLCs and compensators to deliver IMRT [7-11]. This IMRT was the socalled jaws-only IMRT technique (JO-IMRT). With this technique, a series of rectangular fields are delivered from each beam direction to create complex intensity patterns. Most recently, the JO-IMRT is implemented in a commercial system (Panther Treatment planning system, Prowess Inc., Chico, CA) [12]. The introduction of JO-IMRT technique has opened the door for implementing IMRT in developing countries, where the maintenance of the MLC is not near at hand. The long-term objective of our work is to research and apply IMRT using conventional jaws (JO-IMRT). This is deeply meaningful for applying IMRT in our country and other developing country. To achieve our goal, quality assurance (QA) is needed for the IMRT plans before applied to patients. Octavius 4D 1500 (PTW, Freiburg) is an advanced tool enabling the evaluation of plans at all gantry angles. In the previous work, pretreatment patient specific quality assurance for $25 \mathrm{JO}$-IMRT were also performed using Octavius 4D and good agreements were shown $[13,14]$. With simple tools, the plans can be evaluated by measurements with gantry angle set at zero degree. In this report we present the results of the QA for our JO-IMRT plans in 2 different cases: one with the gantry angle set equal to beam 
angle as in the plans (hereafter will be called planning angle), and the other with gantry angle set to zero degree. Gamma index (3\%/3 mm of max dose) was evaluated by comparing the dose measured by the QA phantom and the dose calculated by the Treatment Planning System (TPS). A hypothesis was suggested that the gamma index between 2 groups were different.

\section{Materials and Methods}

The linear accelerator is a Siemens Primus M5497. The TPS was supplied by Prowess (Panther TPS, Prowess Inc.). Twentyfive JO-IMRT treatment plans were selected for this study. For each treatment plan, measurements were done for fields (1) at planning angles and (2) at gantry angle of zero degree, respectively. The comparison of Gamma index between measured dose from Octavius 4D 1500 system (Figure 1) and calculated dose from the TPS were conducted using the software VeriSoft 6.2 (PTW, Freiburg). The Octavius 4D system include a rotation unit, a 2D-array detector plate, an inclinometer, an electrometer and, a control unit. The rotation unit (polystyrene, density of $1.05 \mathrm{~g} / \mathrm{cm}^{3}$ ) is a cylindrical phantom which can rotate around its central axis. The $2 \mathrm{D}$-array detector plate consists 1405 ionization chambers of $0.06 \mathrm{~cm}^{3}$.

The gamma analysis was used with criteria $3 \% / 3 \mathrm{~mm}$ and with threshold of $10 \%$. The average of gamma index for each field condition and the average gamma index difference between 2 field conditions were investigated. Simple paired ttest between the group of (1) fields with planning gantry angle and (2) fields with gantry angle reset to 0 degree was conducted.
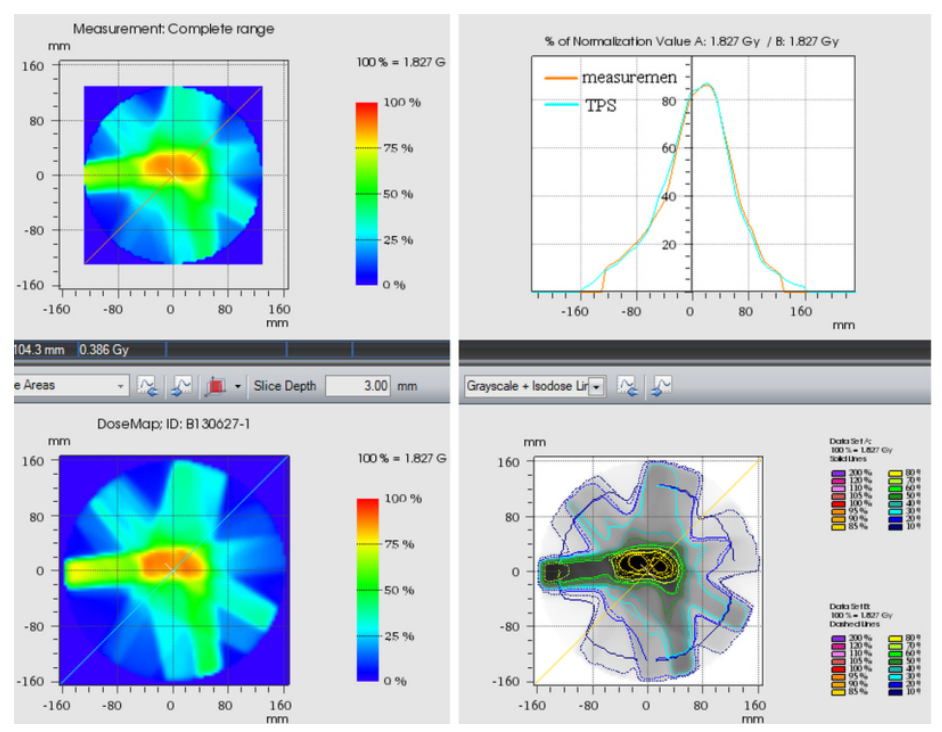

Figure 2. Gamma analysis for planning gantry angles

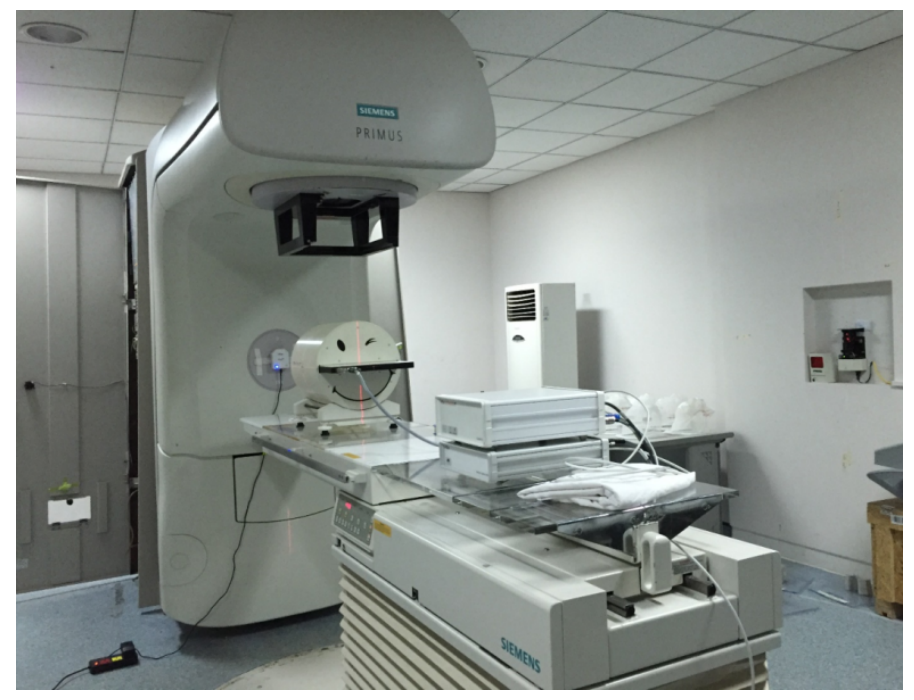

Figure 1. Setup for the pre-treatment quality assurance with Octavius 4D

\section{Results}

The Figures 2 and 3 showed an example of gamma analysis for IMRT delivered with planning angle and zero-degree gantry angle. The gamma indexes of both groups were higher than $90 \%$.

For the fields measured at planning angles and at 0-degree gantry angle, the averaged gamma indexes were $94.72 \pm 2.67 \%$ and $97.32 \pm 2.21 \%$, respectively. The average percentage of gamma passed rate using MapCHECK 2 was $96.77 \pm 2.33 \%$ for 25 JO-IMRT plans in previous studies $[13,14]$. These results show that there is a slight discrepancy between Otavius 4D (using 0-degree gantry angle) and MapCHECK2. Our results are also consistent with the results of researchers $[15,16]$. The averaged gamma index difference between 2 conditions (Figure 4) was $2.59 \pm 2.11 \%(\mathrm{p}<0.0001)$.
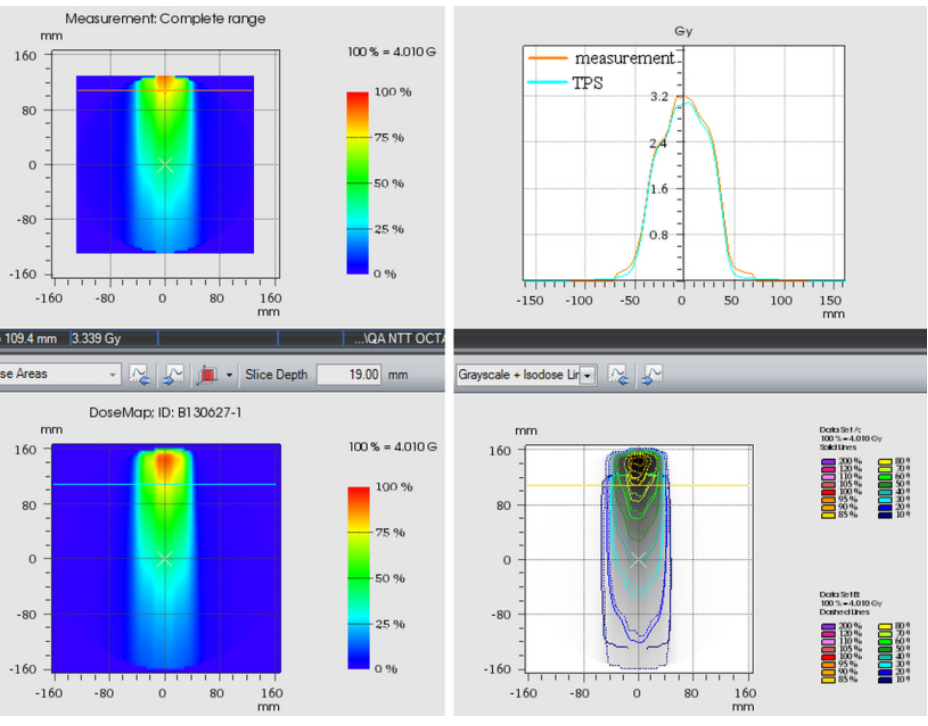

Figure 3. Gamma analysis for 0-degree gantry angle 


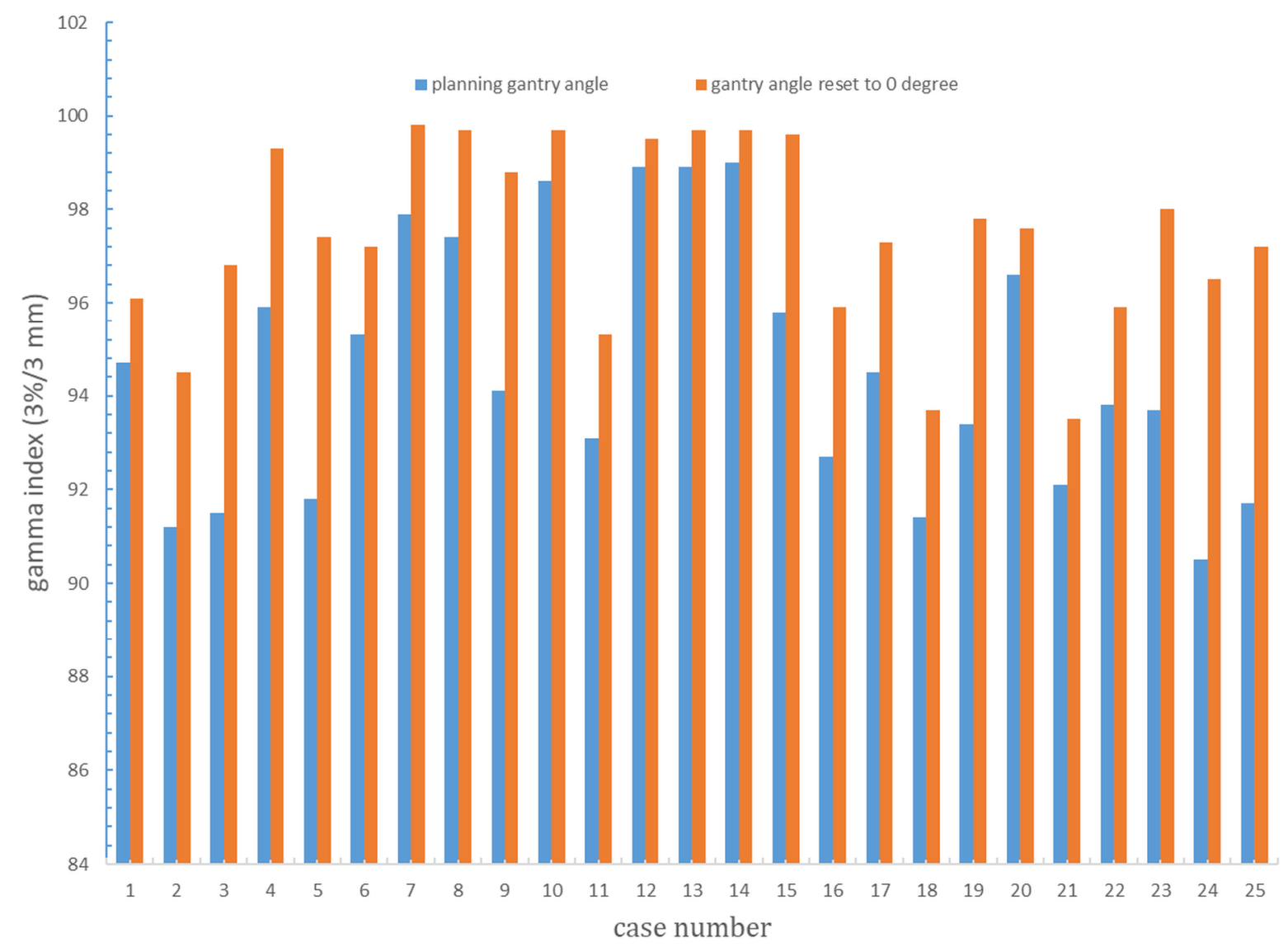

Figura 4. The gamma index with planning gantry angle and gantry angle reset to 0 degree.

\section{Discussion}

The fields measured at planning angle had significantly lower gamma indexes when compared to the fields measured at 0 degree gantry angle. The accuracy of gantry position, the couch insertion in TPS, the directional dependence, and the reproducibility of jaw movement with respected to different gantry angle may contribute to the difference of gamma indexes. For this reason, we see that all the evaluation tests should be performed before the QA process. The OCTAVIUS 4D algorithm requires the PDD measurements and the phantom's density in the TPS. Hence, the commissioning of OCTAVIUS 4D should be the factors that play a vital role in the results of the QA. For the phantom's density in the TPS, the best choice is to set the relative electron density. For PDD commissioning, PDD should be measured in a water phantom at a source-to-surface-distance of $85 \mathrm{~cm}$. In contrast to this, we used simple PDD curves of PTW in the Verisoft application for dose reconstruction which is different from our original PDD data. This variation may contribute to the result of unsatisfactory gamma pass rate. In general, the gamma pass rate is around $97 \%$ for IMRT plan QA.

Although the setting up and measurement at $0^{\circ}$-gantry are much easier than at true gantry angles, the method of measurement at 0-degree gantry angle cannot accurately determine where the error has occurred, nor does it indicate the impact on the actual implementation of the treatment plan. Thus, the best treatment verification should encompass the composite dose distribution under real irradiation conditions. However, the QA results may be influenced by the gravity effect, jaw positional errors, gantry sag, and detectors. Hence before doing IMRT QA with true gantry angles, one ensures that there is no significant variation in LINAC profile characteristics and output due to angular changes to exclude possible deviation. In this study, the test results indicate no angular dependence on gamma value. For QA measurements performed only on fields at gantry angle of zero degree, as an acceptance during the clinical practice, a smaller tolerance should be considered.

\section{Conclusion}

Ideally, all IMRT QA plans should be done at gantry angles equal to beam angles of the treatment plan. Two series of measurements were done for QA of JO-IMRT plans, one at planning angles and one at gantry angle set to zero degree. In both cases, gamma index of more than $90 \%$ were found for all of our 25 JO-IMRT treatment plans. 


\section{Acknowledgment}

A part of this research was presented on proceeding of the 113th Scientific Meeting of the Japan Society of Medical Physics (JSMP) 2017 at the Pacifico Yokohama, Japan.

The first author also would like to extend thanks to PTW company and Mr. Edmund Lo, General Manager at PTW-Asia Pacific Ltd for their sponsors.

\section{Conflict of interest}

The authors declare that they have no conflict of interest.

\section{References}

[1] Webb S. Intensity Modulated Radiation Therapy. Taylor \& Francis Ltd. 2001.

[2] Nishimura Y, Komaki R. Intensity-Modulated Radiation Therapy: Clinical Evidence and Techniques. Springer. 2015; $22-27$.

[3] Webb S. Optimization by simulated annealing of three-dimensional, conformal treatment planning for radiation fields defined by a multileaf collimator: II. Inclusion of two- dimensional modulation of the X-ray intensity. Phys Med Biol. 1992;37(8):1689-1704.

[4] Jeraj M, Robar V. Multileaf collimator in radiotherapy. Radiol Oncol. 2004;38(3):235-240.

[5] Yang J, Li J, Chen L, et al. Dosimetric verification of IMRT treatment planning using Monte Carlo simulations for prostate cancer. Phys Med Biol. 2005;50(5):869-878.

[6] Arnfield MR, Siebers JV, Kim JO, et al. A method for determining multileaf collimator transmission and scatter for dynamic intensity modulated radiotherapy. Med Phys. 2000;27(10):2231-2241.

[7] Earl MA, Afghan MKN, Yu CX, et al. Jaws-only IMRT using direct aperture optimization. Med Phys. 2007;34(1):307-314.

[9] Dai JR, Hu YM. Intensity-modulation radiotherapy using independent collimators: An algorithm study. Med Phys. 1999;26(12):2562-2570.

[10] Shepard DM, Earl MA, Li XA, et al. Direct aperture optimization: A turnkey solution for step-and-shoot IMRT. Med Phys. 2002;29(6):1007-1018.

[11] Mu G, Xia P. A feasibility study of using conventional jaws to deliver complex IMRT plans for head and neck cancer. Phys Med Biol. 2009;54(18):5613-5623.

[12] Nguyen J. Method for intensity modulated radiation treatment using independent collimator jaws. Patent US 71809802006.

[13] Tai DT, Son ND, Loan TTH, Trang NTH. Initial experiences of applying the jaws-only IMRT technique in Dong Nai General Hospital, Vietnam. IFMBE Proceedings. 2018;63:335-339

[14] Tai DT, Son ND, Loan TTH, Anson HPW. Quality assurance of the jaws only-intensity modulated radiation therapy plans for headand-neck cancer. Phys Med. 2017;38:148-152.

[15] Kumar S, Cheruparambil A, Thokkayil AP, et al. Clinically evaluating directional dependence of 2D seven29 ion-chamber array with different IMRT plans. Int J Cancer Ther Oncol. 2015;3(4):348.

[16] Nelms BE, Zhen H, Tomé WA. Per-beam, planar IMRT QA passing rates do not predict clinically relevant patient dose errors. Med Phys. 2011;38(2):1037-1044. 